\title{
Sterile neutrinos: direct mixing effects versus induced mass matrix of active neutrinos
}

\author{
Alexei Yu. Smirnov ${ }^{a, b} 1$ and Renata Zukanovich Funchal ${ }^{c, a}{ }^{2}$ \\ a ICTP, Strada Costiera 11, 34014 Trieste, Italy, \\ ${ }^{b}$ Institute for Nuclear Research, Russian Academy of Sciences, Moscow, Russia \\ ${ }^{c}$ Instituto de Física, Universidade de São Paulo, C. P. 66.318, 05315-970 São Paulo, \\ Brazil
}

\begin{abstract}
Mixing of active neutrinos with sterile ones generate "induced" contributions to the mass matrix of active neutrinos $\sim m_{S} \sin ^{2} \theta_{a S}$, where $m_{S}$ is the Majorana mass of the sterile neutrino and $\theta_{a S}$ is the active-sterile mixing angle. We study possible effects of the induced matrix which can modify substantially the implications of neutrino oscillation results. We have identified the regions of $m_{S}$ and $\sin ^{2} \theta_{a S}$ where the induced matrix (i) provides the dominant structures, (ii) gives the sub-dominant effects and (iii) where its effects can be neglected. The induced matrix can be responsible for peculiar properties of the lepton mixing and neutrino mass spectrum, in particular, it can generate the tri-bimaximal mixing. We update and discuss bounds on the induced masses from laboratory measurements, astrophysics and cosmology. We find that substantial impact of the induced matrix is possible if $m_{S} \sim(0.1-0.3) \mathrm{eV}$ and $\sin ^{2} \theta_{a S} \sim 10^{-3}-10^{-2}$ or $m_{S} \geq 300 \mathrm{MeV}$ and $\sin ^{2} \theta_{a S} \leq 10^{-9}$. The bounds can be relaxed in cosmological scenarios with low reheating temperature, if sterile neutrinos decay sufficiently fast, or their masses change with time.
\end{abstract}

\section{Introduction}

There are two salient properties of neutrinos related to neutrality which distinguish them from other known fermions:

\footnotetext{
${ }^{1}$ smirnov@ictp.it

${ }^{2}$ zukanov@if.usp.br
} 
- the possibility to have a Majorana mass terms;

- mix with new fermions which are singlets of the Standard Model (SM) symmetry group.

So it would seem natural to explain unusual properties of neutrinos, such as smallness of masses and large mixing, using these two features. In fact, the see-saw mechanism [1] employs both.

It may happen however that the see-saw mechanism is not enough to explain the pattern of the lepton mixing, especially if quark-lepton symmetry or unification are imposed. In this connection, we will concentrate on the second feature - the possibility of neutrinos to mix with singlets of the Standard Model, i.e., sterile neutrinos. In general, sterile neutrinos may originate from some other sectors of the theory, e.g. related to supersymmetry breaking or extra dimensions, and not coincide with the right handed components of neutrino fields.

In all, there are two types of effects of the mixing:

- Direct effects - when new states can be produced in various processes and participate in neutrino oscillations, etc.;

- Indirect effects - via the modification of the mass matrix of light active neutrinos.

The role and relevance of these effects is determined by masses and mixings of sterile neutrinos. In some ranges of parameters the cosmological and astrophysical consequences of mixing are more important and the influence on the mass matrix is negligible. In other regions vice versa: direct mixing effects are negligible - mixing becomes "invisible" but the modification of the mass matrix is substantial.

Being light, sterile neutrinos can immediately take part in the phenomenology of neutrino oscillations changing the interpretation of experimental results $[2,3,4]$. Being heavy and weakly mixed, they do not show up in oscillations and other processes, however their "invisible" mixing can strongly modify the mass matrix of active neutrinos and therefore change implications of neutrino results for theory. In particular, in this way, the presence of sterile neutrinos can induce the large or maximal mixing of the active neutrino components $[5,6]$, or, for instance, produce deviation of the 1-2 mixing from maximal [7]. 
In a range of masses $m_{S}<Q$, where $Q$ is the energy release in processes, sterile neutrinos do not decouple since they still can be produced. They can decouple here in the sense that their direct dynamical effects are negligible due to the smallness of mixing. For bigger masses, $m_{S}>Q$, sterile neutrinos decouple, as it happens in the standard see-saw mechanism. This decoupling generates an additional contribution to the mass matrix of active neutrinos and gives negligible deviation from universality.

From observational point of view till now there is no clear evidences of existence of sterile neutrinos, though some hints exist. Those include, the LSND result [8] and its interpretation in terms of oscillations in the $(3+1)$ or $(3+2)$ neutrino mixing schemes; large scale structure formation in the Universe with the warm dark matter composed of the keV sterile neutrinos [9, 10]; high observed velocities of pulsars and their explanation as an asymmetric emission of the keV sterile neutrinos [11]; the early reionization of the universe due to the radiative $S$ - decay [12].

In this paper we study in details the possible effects of sterile-active mixing on the mass matrix of active neutrinos. We obtain bounds on these induced masses from the direct mixing effects. We find the impact of the induced matrix may be considerable if $m_{S} \sim(0.1-0.3) \mathrm{eV}$ and $\sin ^{2} \theta_{a S} \sim 10^{-3}-10^{-2}$ or $m_{S} \geq 300 \mathrm{MeV}$ and $\sin ^{2} \theta_{a S} \leq 10^{-9}$.

The paper is organized as follows. In sec. 2, we determine the mass matrix induced by mixing of active neutrinos with a sterile one. We study the properties of the induced mass matrix and the possibility to explain certain features of the neutrino mass spectrum and mixing pattern using this matrix. We find values of mixing (as a function of the mass of the sterile component) for which the effect of the sterile neutrino (i) explains the dominant structures of the mass matrix; (ii) produces the sub-dominant structures of the mass matrix; (iii) can be neglected, being of the order or below the $1 \sigma$ uncertainties of the present measurements. In sec. 3, we consider various bounds on masses and mixing of the sterile neutrino and consequently, on the induced matrix, in particular those from astrophysics and cosmology. We then, in sec. 4, confront these bounds with regions found in sec. 2 and discuss how they can be improved in the future. We also comment on new physics scenarios which allow to evade the bounds. Our conclusions are given in sec. 5 . 


\section{Active-sterile mixing and induced mass matrix}

\subsection{Induced mass matrix}

Let us consider three active Majorana neutrinos $\nu_{a}=\left(\nu_{e}, \nu_{\mu}, \nu_{\tau}\right)^{T}$ with mass matrix

$$
\mathbf{m}_{\mathbf{a}}=\left(\begin{array}{ccc}
m_{e e} & m_{e \mu} & m_{e \tau} \\
m_{\mu e} & m_{\mu \mu} & m_{\mu \tau} \\
m_{\tau e} & m_{\tau \mu} & m_{\tau \tau}
\end{array}\right)
$$

generated, e.g., by the see-saw mechanism [1]. We consider $m_{a} \leq 1 \mathrm{eV}$ - below the present upper bound to avoid strong cancellations of different contributions.

We assume that (i) the active neutrinos mix with a single (for simplicity) sterile neutrino, $S$, via the masses

$$
m_{a S}^{T} \equiv\left(m_{e S}, m_{\mu S}, m_{\tau S}\right)
$$

(ii) $S$ has a Majorana mass, $m_{S}$, which is much larger than the mixing masses and $m_{a}$ :

$$
m_{S} \gg m_{\alpha S}, m_{a}
$$

So, in the basis $\left(\nu_{a}, S\right)$, the complete mass matrix has the form

$$
\left(\begin{array}{cc}
\mathbf{m}_{\mathbf{a}} & m_{a S} \\
m_{a S}^{T} & m_{S}
\end{array}\right) .
$$

Properties of $S$ : masses, mixing, possible new symmetries, etc., are determined by some new physics which, in general, differs from physics responsible for generation of $\mathbf{m}_{\mathbf{a}}$.

Under condition (3) the block diagonalization gives for the light neutrinos the mass matrix

$$
\mathbf{m}_{\nu} \approx \mathbf{m}_{\mathbf{a}}+\mathbf{m}_{\mathbf{I}}
$$

where

$$
\mathbf{m}_{\mathbf{I}} \equiv-\frac{1}{m_{S}}\left(m_{a S}\right) \times\left(m_{a S}\right)^{T},
$$

is the induced contribution to the neutrino mass matrix due to active - sterile mixing, or shortly, induced mass matrix. For the individual matrix element we have

$$
\left(m_{\nu}\right)_{i j}=\left(m_{a}\right)_{i j}-\frac{m_{i S} m_{j S}}{m_{S}}
$$


Let us introduce the active - sterile mixing angles

$$
\sin \theta_{j S} \approx \frac{m_{j S}}{m_{S}}
$$

Then the induced masses can be written as

$$
\left(m_{I}\right)_{i j}=-\sin \theta_{i S} \sin \theta_{j S} m_{S}
$$

It is this combination of parameters which determines physical effects. For the flavor blind mixing we would have simply the product $\sin ^{2} \theta_{S} m_{S}$.

In the case of a single sterile neutrino the induced contribution is the singular (rank-1) matrix. This feature substantially restricts possible effects of the induced matrix. In the case of two (several) sterile neutrinos, two (several) independent singular contributions to the induced matrix appear:

$$
\mathbf{m}_{\mathbf{I}}=-\sum_{i} \frac{1}{m_{S}^{(i)}}\left(m_{a S}^{(i)}\right) \times\left(m_{a S}^{(i)}\right)^{T}
$$

That opens new possibilities in the description of neutrino mass matrices. Apparently, with three neutrinos any structure of the matrix can be reproduced.

\subsection{Neutrino mass matrix in flavor basis}

To evaluate the impact of the active-sterile mixing, we reconstruct the neutrino mass matrix from the data in the flavor basis in the context of three active neutrinos. The values of matrix elements in terms of the oscillation parameters are given by

$$
m_{\alpha \beta}=m_{1} e^{-i 2 \lambda_{1}} U_{\alpha 1}^{*} U_{\beta 1}^{*}+m_{2} U_{\alpha 2}^{*} U_{\beta 2}^{*}+m_{3} e^{-i 2 \lambda_{3}} U_{\alpha 3}^{*} U_{\beta 3}^{*}
$$

with $\alpha, \beta=e, \mu, \tau$, and $U_{\alpha i}$ being the elements of the PMNS matrix. The matrix elements $U_{\alpha i}$ are functions of the three mixing angles $\theta_{12}, \theta_{13}, \theta_{23}$ and the complex phase $\delta$ given by the standard parametrization of the mixing matrix.

We use the best fit values and $1 \sigma$ intervals of the oscillation parameters from Ref. [13]:

$$
\left|\Delta m_{32}^{2}\right|=2.4\left(1.00_{-0.13}^{+0.11}\right) \times 10^{-3} \mathrm{eV}^{2}
$$




$$
\begin{gathered}
\Delta m_{21}^{2}=7.92(1.00 \pm 0.045) \times 10^{-5} \mathrm{eV}^{2}, \\
\sin ^{2} \theta_{23}=0.44\left(1.00_{-0.11}^{+0.21}\right) \\
\sin ^{2} \theta_{12}=0.314\left(1.00_{-0.075}^{+0.09}\right)
\end{gathered}
$$

and for $\sin ^{2} \theta_{13}$ we take

$$
\sin ^{2} \theta_{13}=0.9\left(1.0_{-0.9}^{+3.1}\right) \times 10^{-2}
$$

with the non-zero best fit value.

The reconstructed matrices for the normal, inverted mass hierarchy and the degenerate mass spectrum are given in Table 1, where we show the absolute values of the matrix elements. For each case we present (i) the matrix for the best fit values of the parameters, (ii) the intervals which correspond to the $1 \sigma$ experimental uncertainties for zero CP-phases and the intervals when also the CP-violating phases vary in whole possible range: $\lambda_{i}=0-\pi$, $\delta=0-\pi / 2$.

The following comments are in order.

1) In the case of normal mass hierarchy we take $m_{1}=0$, so that $m_{2}=\sqrt{\Delta m_{21}^{2}}$ and $m_{3}=\sqrt{\Delta m_{31}^{2}}$. Notice that the 1-3 element of the matrix is much smaller than the 1-2 element and elements of the dominant block are different. This is the consequence of nonzero (though statistically insignificant) 1-3 mixing and shift of the 2-3 mixing from maximal one. The $1 \sigma$ experimental uncertainties lead to $\delta m_{e e} \sim 2.5 \mathrm{meV}, \delta m_{e \mu} \sim \delta m_{e \tau} \sim \delta m_{\mu \tau} \sim 5$ $\mathrm{meV}, \delta m_{\mu \mu} \sim \delta m_{\tau \tau} \sim 10 \mathrm{meV}$. The effect of CP-phases is sub-leading. Variations of phases $\lambda_{3}, \delta$ result in similar size of the intervals and both effects double the indicated uncertainties.

Notice that there are strong correlations between elements, so that their values can not be taken from the indicated intervals independently.

2) For the inverted hierarchy we take $m_{3}=0, m_{1} \approx m_{2} \approx \sqrt{\left|\Delta m_{31}^{2}\right|}$. We choose $\lambda_{1}=\delta=0$ ( $\lambda_{3}$ is irrelevant) for the best fit data analysis. The $1 \sigma$ experimental uncertainties lead to $\delta m \sim(8-10) \mathrm{meV}$ for all matrix elements but $\delta m_{\mu \tau} \sim 4 \mathrm{meV}$. The effect of phase variations is much stronger. Now the phases affect the dominant block and therefore $\delta m \sim m$. 
3) Degenerate spectrum. For illustration we take $m_{1} \approx m_{2} \approx m_{3}=m_{0}=0.2 \mathrm{eV}$. The experimental errors produce very small effect: $\delta m \sim 1 \mathrm{meV}$. In contrast, effects of phase variations are very strong, $\delta m \sim m_{0}$. Here also variations (values) of different elements are strongly correlated.

\subsection{Induced matrix and the dominant structures}

The mass and mixing patterns in quark and lepton sectors are strongly different. The difference can (at least partially) originate from the active-sterile mixing which is absent in the quark sector. The shortcoming of this proposal is the coincidence problem: two different contributions to the mass matrix, active, $\mathbf{m}_{\mathbf{a}}$, and induced, $\mathbf{m}_{\mathbf{I}}$, are of the same order or within 1-2 orders of magnitude in spite of the fact that they have different, and at the first sight, unrelated origins. The only argument in favor is that this will be not the only case we meet the coincidence problem in other areas too. Another possibility is that $\mathbf{m}_{\mathbf{I}} \gg \mathbf{m}_{\mathbf{a}}$.

Let us consider first the case of one sterile neutrino. Due to the singular (rank 1) character of the induced matrix it can not reproduce the dominant structures of the neutrino mass matrix in the case of the degenerate mass spectrum and inverted mass hierarchy. In the former case Det $\mathbf{m} \approx m_{0}^{3}$, where $m_{0}$ is the scale of neutrino mass. In the latter - there are two dominant eigenvalues and the determinant of the 1-2 submatrix is non-zero.

Essentially this means that the matrix induced by one sterile neutrino can be the origin of the dominant block only in the case of normal mass hierarchy. This can explain the large (maximal) 2-3 mixing. Suppose

$$
m_{\mu S}=m_{\tau S}=m_{0}, \quad m_{e S} \ll m_{0},
$$

then

$$
\mathbf{m}_{\mathbf{I}} \approx \frac{m_{0}^{2}}{m_{S}}\left(\begin{array}{ccc}
0 & 0 & 0 \\
0 & 1 & 1 \\
0 & 1 & 1
\end{array}\right) .
$$

From (18) we obtain $2 m_{0}^{2} / m_{S}=\sqrt{\Delta m_{\text {atm }}^{2}}$ or

$$
m_{S} \sin ^{2} \theta_{S}=0.5 \sqrt{\Delta m_{\mathrm{atm}}^{2}} \approx 25 \mathrm{meV} .
$$


Parameters of the 1-2 block (mixing and mass) should be given by the original active mass matrix. For the best fit values of the oscillation parameters (here we take $\sin ^{2} \theta_{13}=0$ ) and the induced matrix (18) the active neutrino mass matrix should be of the form

$$
\mathbf{m}_{\mathbf{a}}=U_{23}^{\mathrm{m}} U_{12}^{\mathrm{sol}} \mathbf{m}_{\mathbf{2}} U_{12}^{\mathrm{solT}} U_{23}^{\mathrm{m} T}=m_{2}\left(\begin{array}{ccc}
s^{2} & \frac{s c}{\sqrt{2}} & -\frac{s c}{\sqrt{2}} \\
\cdots & \frac{c^{2}}{2} & -\frac{c^{2}}{2} \\
\cdots & \cdots & \frac{c^{2}}{2}
\end{array}\right),
$$

where $\mathbf{m}_{\mathbf{2}}=\operatorname{diag}\left(0, m_{2}, 0\right), s \equiv \sin \theta_{12}, c \equiv \cos \theta_{12}, U_{12}^{\text {sol }}$ is the 1-2 rotation matrix on the solar mixing angle, $U_{23}^{\mathrm{m}}$ is the matrix of maximal 2-3 mixing. We assumed that $m_{1} \approx 0$. Notice that all elements of this matrix are nearly equal being in the range $0.31-0.35$.

A non-zero $m_{1}$ would be equivalent of adding to (20) the matrix $m_{1} \mathbf{I}$ proportional to the unit matrix and substituting $m_{2} \rightarrow\left(m_{2}-m_{1}\right)$, if there is no CP-violating phases. That does not change our conclusion provided that $m_{1} \ll m_{2}$.

The induced matrix can be chosen in such a way that the active one has hierarchical structure with small mixings similar to the charged fermion mass matrices. (Partly this case has been studied in [6]). Consider non-universal active-sterile coupling of the type

$$
m_{\alpha S}=\sqrt{\left(m_{3}-m_{1}\right) m_{S}}(\alpha, a, b), \quad \alpha \ll a \sim b .
$$

Then the required mass matrix of active neutrinos can be written as

$$
\mathbf{m}_{\mathbf{a}}=m_{1} \mathbf{I}+\left(m_{3}-m_{1}\right)\left(\begin{array}{ccc}
\epsilon s^{2}-\alpha^{2} & \epsilon \frac{s c}{\sqrt{2}}-\alpha a & -\epsilon \frac{s c}{\sqrt{2}}-\alpha b \\
\ldots & 1-a^{2}+\epsilon \frac{c^{2}}{2} & 1-a b-\epsilon \frac{c^{2}}{2} \\
\ldots & \ldots & 1-b^{2}+\epsilon \frac{c^{2}}{2}
\end{array}\right),
$$

where $\epsilon \equiv m_{2} /\left(m_{3}-m_{1}\right)$. For $a=0.95$ and $b=1.50$ we obtain hierarchical 2-3 block with $\left(m_{a}\right)_{22}:\left(m_{a}\right)_{23}:\left(m_{a}\right)_{33} \approx 0.1: 0.4: 1.2$. Other elements are of the order $0.02-0.04$ if $\alpha \sim 0.01$. Certain hierarchy among those elements can be obtained for non-zero 1-3 mixing and some deviation of the 2-3 mixing from maximal. The overall spread of the values of elements by 2 orders of magnitude can be easily obtained.

Another scenario (essentially considered in [6]) is that $b \gg a$, so that the induced matrix corrects the 33 element only. Taking, e.g., $m_{33}=\sin ^{2} \theta_{\tau S} m_{S} \sim 300 \mathrm{meV}$ and the final 
matrix $\mathbf{m}_{\nu}$ as in the Table 1, we obtain that the original active mass matrix should be like that for normal hierarchy (Table 1 ) but with $m_{33} \sim 300 \mathrm{meV}$, that is, strongly hierarchical.

\subsection{Induced matrix and mass hierarchy}

An interesting possibility is that the induced matrix can switch the mass hierarchy from normal to inverted and vice versa. Indeed,

$$
\mathbf{m}_{\nu}{ }^{\text {inv }} \sim \sqrt{2} \mathbf{m}_{\nu}^{\text {norm }}-\sqrt{\frac{\left|\Delta m_{32}^{2}\right|}{2}} \mathbf{D},
$$

where the induced term, $\mathbf{D}$, is close to the "democratic" matrix with all elements being nearly 1.

\subsection{Induced matrix and tri-bimaximal mixing}

The mass matrix which generates the tri-bimaximal mixing in the normal mass hierarchy case can be presented as

$$
\mathbf{m}=\frac{\sqrt{\left|\Delta m_{32}^{2}\right|}}{2}\left(\begin{array}{ccc}
0 & 0 & 0 \\
0 & 1 & -1 \\
0 & -1 & 1
\end{array}\right)+\frac{\sqrt{\Delta m_{21}^{2}}}{3}\left(\begin{array}{ccc}
1 & 1 & 1 \\
1 & 1 & 1 \\
1 & 1 & 1
\end{array}\right)
$$

It is the sum of two singular matrices. The sub-dominant (second) matrix can be induced by universal mixing with the sterile component

$$
m_{\alpha S}=m_{0}(1,1,1)
$$

Then according to $(24)$

$$
m_{S} \sin ^{2} \theta_{S}=\frac{\sqrt{\Delta m_{21}^{2}}}{3} \approx 3 \mathrm{meV} .
$$

In fact, both matrices in (24) can be induced by the active-sterile mixing, if the second sterile neutrino is introduced with mixing elements

$$
m_{\alpha S}^{\prime}=m_{0}(0,1,-1)
$$

In this case the original active neutrino masses should be very small, e.g., of the order of the Planck mass suppressed scale: $\sim v_{\mathrm{EW}}^{2} / M_{\mathrm{Pl}}$. 


\subsection{Induced matrix and QLC}

If not accidental, the quark-lepton complementarity (QLC) relation [14] can imply that

(i) there is some structure in the lepton sector which generates the bi-maximal mixing $U_{\mathrm{bm}} \equiv U_{23}^{\mathrm{m}} U_{12}^{\mathrm{m}}$; and (ii) there is the quark-lepton symmetry which "propagates" the CKMtype rotations to the lepton sector.

Let us consider a possibility that the induced matrix is responsible for the bi-maximal mixing:

$$
\mathbf{m}_{\mathbf{I}}=\mathbf{m}_{\mathrm{bm}}=U_{\mathrm{bm}} \mathbf{m}^{\mathrm{diag}} U_{\mathrm{bm}}^{T},
$$

whereas the charged lepton mass matrix produces the CKM-type rotation. The original active neutrino matrix should then give very small contribution.

Clearly this scenario can not be realized with only one sterile neutrino: Taking $\mathbf{m}^{\text {diag }}=$ $\operatorname{diag}\left(0,0, m_{3}\right)$ we find that $\mathbf{m}_{\mathbf{I}}=U_{23}^{\mathrm{m}} \mathbf{m}_{\mathbf{3}} U_{23}^{\mathrm{m} T}$ with a single maximal mixing. With two sterile neutrinos the matrix $\mathbf{m}_{\mathbf{b m}}$ which generates the bi-maximal mixing can be reproduced precisely. As an example one can take $m_{\alpha S}=m_{0}(a, b,-b)$ and $m_{\alpha S}^{\prime}=m_{0}(0, x, y)$, where $x=\sqrt{d+0.5 a^{2}-b^{2}}, y=\left(d-0.5 a^{2}+b^{2}\right) / x$, and $a, b, d$ are free parameters.

\subsection{Small and negligible induced contribution}

The induced matrix becomes irrelevant if

$$
\frac{m_{i S} m_{j S}}{m_{S}} \ll\left(m_{a}\right)_{i j}
$$

Let us find conditions under which the effects of sterile neutrino are below the present $1 \sigma$ spread of the matrix elements. Uncertainties depend on the type of mass spectra and are different for different elements.

For the normal mass hierarchy the effects are below $1 \sigma$ if

$$
\sin ^{2} \theta_{e S} m_{S}<2 \mathrm{meV}, \quad \sin ^{2} \theta_{\mu S} m_{S}, \quad \sin ^{2} \theta_{\tau S} m_{S}<5 \mathrm{meV},
$$

For $\mu$ and $\tau$ we have taken the uncertainty of $m_{\mu \tau}$ which is the smallest one. 
For the inverted hierarchy the induced contributions are below $1 \sigma$ uncertainties if

$$
\sin ^{2} \theta_{e S} m_{S}<8 \mathrm{meV}, \quad \sin ^{2} \theta_{\mu S} m_{S}<4 \mathrm{meV} .
$$

Larger effects of sterile neutrinos, $\sin ^{2} \theta_{S} m_{S}<20 \mathrm{meV}$, can be mimicked by the phase variations.

In the case of degenerate spectrum $1 \sigma$ experimental uncertainties restrict

$$
\sin ^{2} \theta_{e S} m_{S}<1 \mathrm{meV} .
$$

The phase change produces the same effect as sterile neutrinos with $\sin ^{2} \theta_{\alpha S} m_{S} \sim 200 \mathrm{meV}$.

Sterile neutrinos can be responsible for fine structures of the mass matrix. The smallest (observable) structure is related to the solar mass split. In the case of inverted hierarchy this would correspond to the contribution $\sin ^{2} \theta_{e S} m_{S} \sim 1.5 \mathrm{meV}$, and for the degenerate mass spectrum we obtain the smallest quantity: $\sin ^{2} \theta_{e S} m_{S} \sim 0.4 \mathrm{meV}$.

So, we can identify three benchmarks:

1) For sterile neutrinos with mixings and masses smaller than

$$
\sin ^{2} \theta_{\alpha S} m_{S}=1 \mathrm{meV}
$$

the effects are below the present $1 \sigma$ experimental uncertainties for the hierarchical spectra. Still these neutrinos can influence the sub-leading structures in the case of the degenerate spectrum.

2) Sterile neutrinos with

$$
\sin ^{2} \theta_{\alpha S} m_{S}=3 \mathrm{meV}
$$

can generate the sub-leading structures in the case of normal mass hierarchy.

3) Sterile neutrinos with

$$
\sin ^{2} \theta_{\alpha S} m_{S}=(20-30) \mathrm{meV}
$$

can generate dominant structures in the case of normal and inverted hierarchies. For larger masses and mixings they can lead to dominant structures of the degenerate spectrum.

In Figs. 1, 2 and 3 we show the lines of constant induced mass $\sin ^{2} \theta_{\alpha S} m_{S}=$ const., in the plane $\sin ^{2} \theta_{\alpha S}$ and $m_{S}$ which correspond to the values in $(33,34,35)$. 


\section{Bounds on the active-sterile mixing}

In this section we describe the direct mixing effects of $S$ and bounds on its masses and mixings.

\subsection{Production, thermalization, decay}

The most stringent bounds follow from astrophysics and cosmology. Sterile neutrinos can be produced in the Early Universe non-thermally through their mixing with active neutrinos, affecting primordial nucleosynthesis, cosmic microwave background radiation as well as the growth of cosmological structures.

We assume that no primordial density of sterile neutrinos existed, and all sterile neutrinos where produced in the Early Universe due to mixing with active neutrinos and oscillations [9, $10]$.

The "thermalization" lines and the "decay" lines in the $m_{S}-\sin ^{2} \theta_{S}$ plane (Figs. 1-3) allow to understand various bounds.

1) The thermalization lines give the lower bounds of the $m_{S}-\sin ^{2} \theta_{S}$ region where the sterile neutrinos are thermalized before the primordial nucleosynthesis. According to Ref. $[15,16]$ the lines are slightly different for mixing with electron and non-electron neutrinos:

$$
\begin{gathered}
m_{S} \sin ^{2} \theta_{S}=0.6 \mathrm{meV}, \quad \text { for } \nu_{\mathrm{e}} \\
m_{S} \sin ^{2} \theta_{S}=0.4 \mathrm{meV}, \quad \text { for } \nu_{\mu}, \nu_{\tau} .
\end{gathered}
$$

Notice that the thermalization lines have the same functional dependence as the isolines of induced mass. Furthermore, the lines are below the benchmarks obtained in $(33,34,35)$. That is, sterile neutrinos with parameters which give significant induced contribution were thermalized in the Early Universe.

2 ) We confront the life time of neutrinos with the time of recombination, $\tau_{\text {rec }} \approx 10^{12}$ $\mathrm{s}$, and the age of the Universe, $\tau_{\mathrm{U}}=4 \times 10^{17} \mathrm{~s}$. The decay rate of $S$ strongly depends on $m_{S}$. For small $m_{S}$ the main channel is $S \rightarrow 3 \nu$ and then the following channels open: 
$S \rightarrow \nu+l+\bar{l}, S \rightarrow \nu+q+\bar{q}$, etc.. The decay rate can be written as

$$
\frac{1}{\tau_{S}} \approx \kappa\left(m_{S}\right) \Gamma_{\mu}\left(\frac{m_{S}}{m_{\mu}}\right)^{5} \sin ^{2} \theta_{S},
$$

where $m_{\mu}$ and $\Gamma_{\mu}$ are the muon mass and decay rate, correspondingly, and $\kappa\left(m_{S}\right)$ is the number of decay channels for a given value of $m_{S}$. So, for the non-relativistic sterile neutrinos, the lines of constant decay rate are given by

$$
\kappa\left(m_{S}\right) m_{S}^{5} \sin ^{2} \theta_{S}=\text { const. }
$$

In Figs. 1, 2 and 3 we show two decay isolines corresponding to $\tau_{S}=\tau_{\text {rec }}$ and $\tau_{S}=\tau_{U}$.

\subsection{LSS formation bound}

If $\tau_{S}>\tau_{U}$, the sterile neutrinos contribute to the dark matter in the Universe. Analysis of the Large Scale Structure (LSS) of the Universe gives the bound on the total energy density in sterile neutrinos, $\rho_{S}$, as function of its mass:

$$
\omega_{S} \equiv \frac{\rho_{S}}{\rho_{\mathrm{cr}}} h^{2} \leq \omega_{S}\left(m_{S}\right)
$$

Here $\rho_{\text {cr }}$ is the critical energy density and $h$ is the Hubble constant ${ }^{3}$. We use for $\omega_{S}\left(m_{S}\right)$ the results of the analysis in [17] and [18].

For $m_{S}<100 \mathrm{eV}, S$ compose the hot dark matter component and the bound on $\omega_{S}$ is stronger. According to [17] for $m_{S}<30 \mathrm{eV}$ one has $\omega_{S}<0.005$ at 95\% CL. The bound weakens with the increase of $m_{S}$, as neutrinos become colder: $\omega_{S}<0.02$ at $m_{S}=100 \mathrm{eV}$, and $\omega_{S}<0.12$ at $m_{S}=300 \mathrm{eV}$. In the interval $m_{S}=(0.25-30) \mathrm{eV}$ the $95 \% \mathrm{CL}$ bound from [18] can be parametrized as

$$
\omega_{S} \leq 0.001\left[\left(\ln \left(\frac{m_{S}}{\mathrm{eV}}\right)-1.7\right)^{2}+2.5\right] .
$$

\footnotetext{
${ }^{3}$ In this paper in numerical estimations we use $\mathrm{h}=0.7$.
} 
For $m_{S}=(30-300) \mathrm{eV}^{4}$ :

$$
\omega_{S} \leq 0.01\left[\left(\ln \left(\frac{m_{S}}{\mathrm{eV}}\right)-2.55\right)^{2.2}-1.0\right]
$$

and $\omega_{S}<0.12$ for $m_{S}>300 \mathrm{eV}$.

Calculations of the energy density $\omega_{S}$ have been updated recently [19] with inclusion of a number of additional effects, in particular, effects of the quark-hadron transition, modification of the finite temperature effective mass of the active neutrinos, etc.. For the temperature of the quarks-hadron transition $T_{Q C D}=170 \mathrm{MeV}$, the relation between $\omega_{S}, m_{S}$ and $\theta_{S}$ can be parametrized as [19]

$$
m_{S}=1.45 \mathrm{keV}\left(\frac{10^{-8}}{\sin ^{2} \theta_{S}}\right)^{0.615}\left(\frac{\omega_{S}\left(m_{S}\right)}{0.13}\right)^{0.5},
$$

or

$$
\sin ^{2} \theta_{S}=1.83 \cdot 10^{-8}\left(\frac{\omega_{S}\left(m_{S}\right)}{0.13}\right)^{0.813}\left(\frac{1 \mathrm{keV}}{m_{S}}\right)^{1.626} .
$$

Notice that for $m_{S}<3 \mathrm{keV}$ it deviates from the dependence previously found in [20].

Plugging the limits (41) and (42) in relation (44) we find the upper bound on $\sin ^{2} \theta_{S}$ as a function of $m_{S}$ (see region LSS in Figs. 1-3). The bound is absent for $m_{S}<0.25 \mathrm{eV}$ [21]. It does not depend on the flavor of active neutrino to which sterile neutrino mixes.

The bound is valid for the region of parameters below the isoline $\tau_{S}=\tau_{U}$ which corresponds to $m_{S}<0.5 \mathrm{MeV}$ and $\sin ^{2} \theta_{S}>7 \cdot 10^{-13}$. However for $m_{S}>5 \mathrm{keV}$ the stronger bound follows from the diffuse background radiation.

It has been shown in a recent analysis [22] that sterile neutrinos with mass $m_{S}<14$ $\mathrm{keV}$ are excluded at 95\% CL as the dark matter particles responsible for the LSS formation. This implies somehow stronger bound on $\omega_{S}$ than the one we use in our estimations and consequently stronger bound on the $S$ parameters. It is argued in that the bound can be relaxed if new particle decays in the epoch between the decoupling of $S$ and BBN increase the

\footnotetext{
${ }^{4} \mathrm{~A}$ more accurate parametrization in this range is: $\omega_{S} \leq 6 \cdot 10^{-3}+4.1 \cdot 10^{-6}\left(m_{S} / \mathrm{eV}\right)+2.5 \cdot 10^{-7}\left(m_{S} / \mathrm{eV}\right)^{2}+1.7 \cdot 10^{-8}\left(m_{S} / \mathrm{eV}\right)^{3}-4.5 \cdot 10^{-11}\left(m_{S} / \mathrm{eV}\right)^{4}$
} 
entropy, thus diluting concentration of $S$ and reducing their relative temperature [23]. This may be the case for the model proposed in $[24,25]$ where two heavy RH have masses above $1 \mathrm{GeV}$. (Explanation of LSS by $S$ would probably require that $S$ are produced at some high energy scales by processes that are not related to active-sterile neutrino oscillations [23].)

\subsection{Limit from cosmic X-ray radiation}

Due to mixing via the loop diagrams sterile neutrinos decay into an active neutrino and a photon: $S \rightarrow \nu_{a} \gamma$ with $E_{\gamma} \approx m_{S} / 2$ and at the rate $\Gamma_{\gamma} \sim \alpha \Gamma_{3 \nu}$. Therefore one expects to detect the photon emission line when looking at big concentrations of dark matter such as galaxy clusters.

The analyzes of the X-ray emission from the Virgo cluster [26, 27] give limits on the decay and therefore on the mixing of sterile neutrino: $\sin ^{2} \theta_{S}<2.6 \times 10^{-6}\left(m_{S} / \mathrm{keV}\right)^{-4}$. (This parametrization is valid for $m_{S}=(1-10) \mathrm{keV}$.) It is argued in [28] that the bound from Virgo is about 1 - 2 orders of magnitude weaker especially for low masses, $m_{S}<(5-8)$ $\mathrm{MeV}$. However for $m_{S}=10 \mathrm{keV}$, relevant for this analysis (where X-ray bounds start to dominate over the LSS one), the bounds are comparable: $\sin ^{2} \theta_{S}<2.6 \times 10^{-10}$ [26] and $\sin ^{2} \theta_{S}<5.0 \times 10^{-10}[28]$. At the same time stronger limit has been obtained from analysis of Coma cluster [28]:

$$
\sin ^{2} \theta_{S}<2 \times 10^{-5}\left(\frac{m_{S}}{\mathrm{keV}}\right)^{-5} .
$$

The $\gamma$-flux from all possible sources could accumulate over the history of the Universe and be seen as a Diffuse Extragalactic Background RAdiation (DEBRA). Apparently the flux from the radiative $S$ decay should be smaller than the observed flux. In the range $m_{S}=(1-100) \mathrm{keV}$ the DEBRA limit can be parametrized as [29]

$$
\sin ^{2} \theta_{S}<3.1 \times 10^{-5}\left(\frac{m_{S}}{\mathrm{keV}}\right)^{-5}
$$

This bound based on the data collected from the whole sky is weaker than the one given in (45). However, it does not depend on assumptions concerning clustering and therefore is considered to be more robust [29]. These limits are valid below the recombination line $\tau_{S}=\tau_{\text {rec }}$ 
The X-ray exclusion region shown in Figs. 1-3 has been obtained from the analysis of Coma cluster data as well as data on diffuse X-ray background (DEBRA) from HEAO-1 and XMM-Newton missions in [29]. We have also included the very recent bound from the diffuse X-ray spectrum of the Andromeda galaxy [30]. This is the most stringent limit in the range $m_{S}=(1-24) \mathrm{keV}$. For small masses, $m_{S} \lesssim 1 \mathrm{keV}$, we use the limit from [31].

Note in Figs. 1-3 that the X-ray data reduces the parameter space allowed for Warm Dark Matter to a very small region: $m_{s}=(1.7-3.5) \mathrm{keV}$ for $\sin ^{2} \theta_{S} \sim 10^{-9}-10^{-8}$. Also most of the parameter region that can explain the origin of pulsar velocities is ruled out, remaining only a corner around $m_{S} \sim 2-4 \mathrm{keV}$.

It was proposed in Ref. [26] to observe clusters of galaxies with Chandra and XMMNewton observatories, in their high sensitivity range for X-ray photon detection of (1 10) $\mathrm{keV}$. That will allow one to set the limit in the range $10^{-13}<\sin ^{2} 2 \theta_{S}<10^{-5}$ for $m_{S}=(0.6-40) \mathrm{keV}$.

\subsection{CMB-bound}

If the sterile neutrinos decay producing light neutrinos between the active neutrino decoupling time, $\tau_{\nu} \sim 1 \mathrm{~s}$, and the photon decoupling time, $\tau_{\text {rec }}$, this would increase the energy density of relativistic particles at $t \lesssim \tau_{\text {rec }}$. The density is described by the effective number of neutrinos, $N_{\nu}$. That, in turn, affects the CMB angular power spectrum (acoustic peaks). The bounds on $N_{\nu}$ from observations were substantially improved during the last 5 years: $N_{\nu}<13$ at 95\% CL (BOOMERanG/MAXIMA) [32], $N_{\nu}<8.3$ at 90\% CL (WMAP data) [33], $N_{\nu}<6.8-7.1$ at 95\% CL (WMAP and LSS) [34, 35, 36], $N_{\nu}<5.4$ at $95 \% \mathrm{CL}$ (WMAP, LSS and type Ia supernova data) [37].

With the help of considerations in Ref. [38] and [20], we get the "CMB" limit on the sterile neutrino parameters as a function of $N_{\nu}$, which can be parametrized as

$$
\left(\frac{m_{S}}{\mathrm{keV}}\right)^{4}=3 \times 10^{33}\left(N_{\nu}-3\right)^{-2.87} \sin ^{2} \theta_{S}
$$

Combined analysis of the cosmological data on LSS, supernovas and the CMB including 3 
years result from WMAP [39] allows to put the bound $N_{\nu}<3.74$ at 95\% CL. Using this results and Eq. (47) we find the bound shown in Figs. 1-3. This limit is not valid above the line $\tau_{S}<\tau_{\nu} \sim 1 \mathrm{~s}$, which for $\sin ^{2} \theta_{S}=10^{-12}$ corresponds to $m_{S}=(400-500) \mathrm{MeV}$.

In the future, the PLANCK mission $[40,41]$ will allow to strengthen the bound down to $N_{\nu}<3.2$, while according to Ref. [41], the CMBPOL mission can achieve $N_{\nu}<3.05$. This will further expand the excluded region, in particular to larger values of $m_{S}$.

\subsection{BBN bound}

Apparently for one sterile neutrino the limit on the effective number of additional degrees of freedom during the epoch of Big Bang Nucleosynthesis (BBN), $\Delta N_{\nu}=1$, does not provide any bound since at most the equilibrium concentration of $S$ can be produced in the scenarios under consideration. The limit $\Delta N_{\nu} \leq 1$ becomes relevant in the case of more than two sterile states.

For the low values of $m_{S}$ we use the limits from [42] which for $\Delta N_{\nu}=1$ can be parametrized as

$$
\begin{gathered}
m_{S} \sin ^{2} \theta_{S}=1.4 \mathrm{meV}, \text { for } \nu_{e} \\
m_{S} \sin ^{2} \theta_{S}=1.0 \mathrm{meV}, \quad \text { for } \nu_{\mu}, \nu_{\tau} .
\end{gathered}
$$

For the high masses, $m_{S}=10-200 \mathrm{MeV}$, the exclusion region has the shape of a parallelogram [43]. The right boundary is basically given by

$$
\sin ^{2} \theta_{S}\left(\frac{m_{S}}{\mathrm{keV}}\right)^{3}=1.25 \cdot 10^{11}
$$

which is the condition for the heavy neutrinos to be relativistic at decoupling so their number density is not Boltzmann suppressed and they can have an impact on BBN. The left boundary,

$$
\left(\frac{m_{S}}{\mathrm{keV}}\right)^{2} \sin ^{2} \theta_{S}<6 \times 10^{-2}
$$

can be simply understood as the condition that the energy density of the sterile neutrino is smaller (including also an entropy dilution factor 5) than the energy density of one light neutrino species at BBN. 


\subsection{Supernova neutrino bound}

Two different bounds follow from observation of the antineutrino signal from SN1987A [44]. One is from $\bar{\nu}_{e}$-disappearance and the other from star cooling.

1) The resonance conversion $\bar{\nu}_{e} \rightarrow \bar{S}$ can occur in the central regions of the star due to change of sign of the matter potential. That happens for the active-sterile system when the relative electron number density is $Y_{e} \sim 1 / 3$. For the mass range $m_{S} \sim(1-100) \mathrm{eV}$ the adiabaticity condition can be fulfilled if $\sin ^{2} \theta_{S}>10^{-5}$. The adiabatic conversion leads to strong suppression of the $\bar{\nu}_{e^{-}}$flux [3]. So, the observation of the $\bar{\nu}_{e^{-}}$signal from SN1987A gives the bound on the oscillation parameters. In Figs. 1-2 we show the updated results obtained in Ref. [4].

2) For large masses, $m_{S}$, the $\left(\nu_{a} \rightarrow S\right)$ oscillations as well as scattering lead to production of sterile neutrinos in the core of the collapsing star. If these neutrinos escape the core, they can lead to substantial energy loss. This, in turn, will shorten the neutrino burst and the energy released in $\bar{\nu}_{e}$ will be smaller $[31,45]$.

Normal duration of the SN1987A neutrino burst excludes significant cooling effect which puts the bound on $S$ parameters [43] shown in Figs. 1-3. Notice that the upper bound, $\sin ^{2} \theta_{S} \lesssim 10^{-10}$, is slightly different for $\nu_{e}$ and $\nu_{\mu}$ and $\nu_{\tau}$. If mixing is sufficiently large, the sterile neutrinos will be trapped inside the core, and the energy-loss argument is not applicable. This gives the lower bound of the excluded region $\sin ^{2} \theta_{S} \lesssim 10^{-2}$. The mass range is restricted by the condition that $S$ is produced inside the core.

Future detection of high statistics neutrino signal from a Galactic supernova will allow to put stronger bounds. Indeed, another resonance in $\nu_{e}-S$ can occur in the outer regions of the star with normal chemical composition. The adiabaticity condition can be written as

$$
\gamma=10^{2} \sin ^{2} \theta_{S}\left(\frac{m_{S}}{10^{-3} \mathrm{keV}}\right)^{3.3} \gg 1
$$

which in fact is stronger than the cosmological bound in the range $(0.01-0.1) \mathrm{keV}$. The adiabatic $\nu_{e}-S$ conversion leads to the disappearance of the $\nu_{e}$-neutronization peak, and the modification of the signal during the cooling stage. In particular, in the case of normal mass hierarchy the electron neutrino flux at the Earth will be $F\left(\nu_{e}\right)=F^{0}\left(\nu_{\mu}\right)$ in the case 
of large 1-3 mixing and $F\left(\nu_{e}\right)=\cos ^{2} \theta_{12} F^{0}\left(\nu_{\mu}\right)$ for very small 1-3 mixing [3].

In the $\mathrm{eV}$ mass range, sterile neutrinos can drive non negligible $\nu_{\mu} \rightarrow \nu_{e} / \bar{\nu}_{\mu} \rightarrow \bar{\nu}_{e}, \bar{\nu}_{\tau}$ conversions at $\mathrm{TeV}$ energies by the MSW effect that can be constrained by future IceCube data $[46]$.

\subsection{Laboratory bounds}

The laboratory bounds are typically much weaker than the astrophysical and cosmological ones. They are, however, more robust and turn out to be the main bounds if, for some reason (see sec. 4.2), the cosmological and astrophysical limits become inapplicable.

1). The $0 \nu \beta \beta$-decay. Introduction of active-sterile mixing (described by $m_{\alpha S}$ ) does not change the $m_{e e}$ element of the whole $4 \times 4$ mass matrix (4). Therefore for light $S$ : $m_{S} \ll 1 / r_{N}$, where $r_{N}$ is the typical size of nuclei, the rate of $0 \nu \beta \beta$-decay in the lowest approximation is determined by $m_{e e}$ and the effect of $S$ is strongly suppressed.

However, the effect of $S$ on the $0 \nu \beta \beta$-decay increases with $m_{S}$. Let us consider this in more details. The $0 \nu \beta \beta$ decay amplitude, $A^{(S)}$, has two contributions associated to $S$ : i) from the induced mass, $\left(m_{I}\right)_{e e}$, and exchange of light neutrinos, and ii) from exchange of $S$ and its mixing with $\nu_{e}$. So, the amplitude of the decay can be written as

$$
A^{(S)} \propto \frac{\left(m_{I}\right)_{e e}}{\bar{q}^{2}-m_{\nu}^{2}}+\frac{m_{S} \sin ^{2} \theta_{e S}}{\bar{q}^{2}-m_{S}^{2}},
$$

where $\bar{q} \sim 1 / r_{N}$ is the effective momentum of the exchanged neutrino. As it can be inferred from the calculation presented in [47], $\bar{q} \approx 100 \mathrm{MeV}$. In the denominator of (51) the parameter $m_{\nu}$ is the effective mass of light neutrinos and since $m_{\nu} \ll m_{S}$ it can be neglected. Taking into account that the induced mass $\left(m_{I}\right)_{e e}=-m_{S} \sin ^{2} \theta_{e S}$ we can write, according to Eq. (51)), the total contribution of $S$ to the effective Majorana mass as

$$
m_{e e}^{(S)}=m_{S} \sin ^{2} \theta_{e S}\left|1-\frac{\bar{q}^{2}}{\bar{q}^{2}-m_{S}^{2}}\right| .
$$

If $m_{S}$ is negligible the two contributions cancel each other leading to zero effect of $S$. If $m_{S}^{2} \ll \bar{q}^{2}$, we find from $(52)$

$$
m_{e e}^{(S)}=\sin ^{2} \theta_{e S} \frac{m_{S}^{3}}{\bar{q}^{2}} .
$$


So, for light $S, m_{S} \ll 1 / r_{N}, m_{e e}^{(S)} \rightarrow 0$ and the total effective Majorana mass is determined by the ee-elements of the active neutrino mass matrix $\left(\mathbf{m}_{\mathbf{a}}\right)_{e e}$, in agreement with consideration in terms of $4 \times 4$ matrix.

For $m_{S}^{2} \gg \bar{q}^{2}$, the second term in (52) can be neglected and the Majorana mass is given by the induced contribution (as in the usual see-saw mechanism):

$$
m_{e e}^{(S)}=\sin ^{2} \theta_{e S} m_{S}
$$

In Fig. 1 we show the excluded region of the $S$ parameters which corresponds to the upper bound $m_{e e}<0.5 \mathrm{eV}$ obtained from studies of $0 \nu \beta \beta$-decay of ${ }^{76} \mathrm{Ge}$ [48]. We assume that there is no cancellation between $S$ contribution and $\left(\mathbf{m}_{\mathbf{a}}\right)_{e e}$. In general, cancellation reduces the excluded region. However, in our context, the cancellation can not be strong. Indeed, according to our assumption (Sec. 2.1) the original active neutrino mass terms are below 1 $\mathrm{eV}$. Therefore maximal contribution to the effective Majorana mass of electron neutrino is about $1 \mathrm{eV}$, and consequently, only the order of $1 \mathrm{eV}$ contribution from new neutrino state can be cancelled. That corresponds to the long-dashed line in Fig 1.

As follows from Fig. 1 the double beta decay limit becomes relevant for high masses $m_{S}>100 \mathrm{MeV}$.

Future neutrinoless double beta decay experiments [49] with sensitivity down to $m_{e e}<$ $(0.01-0.03) \mathrm{eV}$ will improve the bound on $\sin ^{2} \theta_{S}$ by a factor $10-30$.

2) The $\beta$-decay. The region $m_{S} \sim\left(0.1-10^{3}\right) \mathrm{keV}$ and $\sin ^{2} \theta_{S} \lesssim 10^{-3}-10^{-2}$ for $\nu_{S}-\nu_{e}$ mixing (Fig. 1) is excluded by the negative results of searches of kinks in the energy spectra of nuclear $\beta$-decays [50].

3) Meson decays can have contributions from sterile neutrinos which can modify the energy spectra of their decay products. In the $\nu_{S}-\nu_{e}$ channel, the best limit comes from precision measurements of the energy spectrum of $e^{+}$in the decay $\pi^{+} \rightarrow e^{+} \nu_{e}[51]: \sin ^{2} \theta_{S}<$ $10^{-7}$ for $m_{S}=(50-130) \mathrm{MeV}$. In the $\nu_{S}-\nu_{\mu}$ channel, the bounds come from studies of the spectra of $\pi^{ \pm}$and $K^{ \pm}$decays at accelerators and in the atmosphere. The $90 \%$ CL excluded 
region (labeled as "Decays" in Fig. 2) is taken from Ref. [52]. It is the only bound which can compete with the cosmological and astrophysical limits in the range of large masses $m_{S} \sim(0.03-0.3) \mathrm{GeV}$.

In the near future, the studies of pion, muon and kaon decays at MiniBooNE [53], MINOS [54] and K2K [55] experiments are expected to reach sensitivities of a few $\times 10^{-7}$ or less for the mixing if $m_{S} \sim 100 \mathrm{MeV}$ [52].

Oscillations of active to sterile neutrinos lead to the suppression of the neutral current interaction rate. In this connection, it has been proposed to study interactions of neutrinos from pion decays at rest with the superallowed neutral current reaction $\nu_{x}{ }^{12} \mathrm{C} \rightarrow \nu_{x}{ }^{12} \mathrm{C}^{*}[56]$. The sensitivity of such an experiment can reach $\sin ^{2} \theta_{S} \sim 10^{-2}$ for $m_{S} \sim 1 \mathrm{eV}$.

4) The atmospheric neutrinos and K2K. The combined analysis of the SuperKamiokande [57] and MACRO [58] results as well as the data from accelerator experiment K2K leads to the following $90 \% \mathrm{CL}$. bounds [4]: for the $\nu_{e}-\nu_{S}$ channel $\sin ^{2} \theta_{S}<10^{-2}$, in the interval $m_{S}=(0.01-0.1) \mathrm{eV}$ (see Fig. 1); for the $\nu_{\mu}-\nu_{S}$ channel $\sin ^{2} \theta_{S}<0.04$ in the range $m_{S}=(0.1-1) \mathrm{eV}$ (see Fig. 2); for the $\nu_{\tau}-\nu_{S}$ mixing $\sin ^{2} \theta_{S}<0.1$.

If the cosmological bound is invalid, the atmospheric bound becomes also relevant for larger masses, $m_{S}=(1-10) \mathrm{eV}$.

Sterile neutrino oscillations for $m_{S} \sim$ few eV have the oscillation length comparable to the Earth's radius at $E_{\nu} \sim 1 \mathrm{TeV}$. In the future, IceCube [59] detection of atmospheric neutrinos can probably bring the limit on $\nu_{e}-\nu_{S}$ mixing down to $\sim 10^{-2}$ for $m_{S} \sim$ few $\mathrm{eV}[46]$.

It has been suggested in Ref. [60] that showers generated by ultra-high energy sterile neutrinos $\left(E_{\nu} \sim 10^{6}-10^{12} \mathrm{GeV}\right)$ with $\sin ^{2} \theta_{S} \sim 0.01-0.1$, may be distinguished from the ones generated by active neutrinos in experiments such as EUSO [61] or OWL [62], using the Earth as a filter, as proposed in Ref. [63] for neutralino showers. The mass range of $m_{S}$ that can be tested by these experiments is determined by the mechanism of their production.

5) Reactor neutrino experiments can set limits on the mixing $\bar{\nu}_{e}-\bar{\nu}_{S}$ by comparing 
expected with observed $\bar{\nu}_{e}$ flux. Bugey [64], CHOOZ [65] and Palo Verde [66] provide the main bound on $\bar{\nu}_{e}-\bar{\nu}_{S}$ mixing, $\sin ^{2} \theta_{S} \lesssim 0.01$ (90\% CL.), in the range $m_{S}=(0.3-1) \mathrm{eV}$.

The forthcoming reactor experiment Double-CHOOZ and the proposed projects Kaska, Braidwood and Angra [67], may be able to reach $\sin ^{2} \theta_{S} \sim 5 \cdot 10^{-3}$ for $m_{S}=(0.03-0.7)$ $\mathrm{eV}[68]$.

6) Accelerator bounds. The accelerator oscillation experiments CDHS [69], CCFR [70], NOMAD [71] and CHORUS [72] as well as LSND [8], KARMEN [73], provide with the bound on $\nu_{\mu}-S$ mixing in the range low mass range, $m_{S}>(1-100) \mathrm{eV}$. In particular, $\sin ^{2} \theta_{S}<7 \cdot 10^{-3}(90 \% \mathrm{CL})$ for $m_{S}>10 \mathrm{eV}$ (see region labeled "Beam" in Fig.2).

In Figs. 1 we show the forbidden region of the parameters (labeled "Reac.+Beam") from the combined analysis of these accelerator beam and reactor experiments Ref. [4]. This result excludes the induced mass as the origin of the dominant structure in the low $m_{S}$ domain.

In the high mass range, $m_{S}>0.1 \mathrm{GeV}$ mixing with a heavy $S$ leads to an effective violation of lepton universality and appearance of the flavor changing neutral currents. The non-observation of these effects in experimental data permitted the authors of Ref. [74] to set the 90\% CL limits, shown in Figs. 1-3: $\sin ^{2} \theta_{S}<7.1 \cdot 10^{-3}\left(\nu_{S}-\nu_{e}\right)$, valid in the range $m_{S}>0.14 \mathrm{GeV}\left(m_{\pi}\right), \sin ^{2} \theta_{S}<1.4 \cdot 10^{-3}\left(\nu_{S}-\nu_{\mu}\right)$, valid in the range $m_{S}>1.115 \mathrm{GeV}$ $\left(m_{\Lambda}\right)$, and $\sin ^{2} \theta_{S}<1.7 \cdot 10^{-2}\left(\nu_{S}-\nu_{\tau}\right)$, valid in the range $m_{S}>1.777 \mathrm{GeV}\left(m_{\tau}\right)$. Also searches for a singlet neutral heavy lepton at LEP [75], have allowed to exclude $\sin ^{2} \theta_{S}$ down to $10^{-4}-10^{-1}$ depending on the channel, in the range $0.4<m_{S} / \mathrm{GeV}<90$ at $95 \% \mathrm{CL}$.

Accelerator experiments sensitive to oscillations with $\Delta m^{2}>\Delta m_{32}^{2}$ will be able to set stringent bounds in the low mass range. The MINOS detector [54] can use the neutral current to charged current ratio to probe $\nu_{\mu}-\nu_{S}$ and $\nu_{\tau}-\nu_{S}$ mixing down to $\sin ^{2} \theta_{S} \sim$ $10^{-2}$ [4]. Sensitivity to $\nu_{\mu}-\nu_{S}$ mixing at the level $\sin ^{2} \theta_{S} \sim 10^{-3}$ for $m_{S}=(0.03-0.3) \mathrm{eV}$ can be reached by $\mathrm{T} 2 \mathrm{~K}[76], \mathrm{NO} \nu \mathrm{A}[77]$ and future $\nu$-factories looking for $\nu_{\mu}, \bar{\nu}_{\mu}$ disappearance.

Forthcoming $p p$ and planned $e^{+} e^{-}$collider experiments can expand the the excluded region of $S$ parameters to larger masses. According to Ref. [78], the search for same-sign 
dilepton production mediated by a sterile neutrino $S$ in $p p \rightarrow l^{+} l^{\prime} X$ with $l, l^{\prime}=e, \mu, \tau$, may be used to constrain $\sin ^{2} \theta_{S} \lesssim 10^{-2}$ for $m_{S}=(0.1-2) \mathrm{TeV}$ at the Large Hadron Collider at CERN, provided a large integrated luminosity becomes available. It has been shown [79] that at an International Linear Collider with a center of mass energy $\sqrt{s}=500 \mathrm{GeV}$, one can look for single heavy $S$ production through $e^{+} e^{-} \rightarrow S \nu \rightarrow l W \nu$ with $l=e, \mu, \tau$. This has a sensitivity down to $\sin ^{2} \theta_{S}<7 \cdot 10^{-3}$ for $m_{S}=(200-400) \mathrm{GeV}$. The same reaction at a future Compact Linear Collider operating with $\sqrt{s}=3 \mathrm{TeV}$ could be used to limit $\sin ^{2} \theta_{S}<(2-6) \cdot 10^{-3}$ for $m_{S}=1-2 \mathrm{TeV}[80]$.

Notice however, that these high mass bounds are essentially irrelevant in our context. Indeed, for $m_{S}>1 \mathrm{GeV}$ and $\sin ^{2} \theta_{S}>10^{-3}$, the induced mass is $m_{I}>1 \mathrm{MeV}$. This means that the elements of the original active neutrino mass matrix should also be large $m_{a}>1 \mathrm{MeV}$, and it should be extremely strong cancellation of the original and induced contributions to obtain phenomenologically acceptable masses of light neutrinos: $\left(\left(\mathbf{m}_{\mathbf{a}}\right)_{i j}-\right.$ $\left.\left(\mathbf{m}_{\mathbf{I}}\right)_{i j}\right) /\left(\left(\mathbf{m}_{\mathbf{a}}\right)_{i j}<10^{-7}\right.$.

Inversely, discovery of the $S$ with relatively large mixing in high energy collisions will testify against the approach developed in this paper.

\section{Induced mass versus direct mixing effects}

\subsection{Bounds on induced mass}

Confronting the lines of constant induced masses (33), (34) and (35) with cosmological astrophysical and laboratory bounds in the $\sin ^{2} \theta_{S}-m_{S}$ plane we can conclude on the relative importance of the direct and indirect effects.

There are two regions of parameters in the $\sin ^{2} \theta_{S}-m_{S}$ plane, where the induced masses are more important than the effects of direct mixing. That is, in these regions substantial induced masses for the active neutrino mass matrix are not excluded by the existing bounds.

1) High mass region: $m_{S} \gtrsim 300 \mathrm{MeV}$ and $\sin ^{2} \theta_{S} \lesssim 10^{-9}$. This region is restricted essentially by the CMB bound, meson decays and SN1987A cooling. Future measurements 
can probably improve the bounds by about 1 order of magnitude from below.

Only for the $\nu_{e}-\nu_{S}$ channel, the neutrinoless double beta decay can probe the whole high mass region. Present bounds correspond essentially to the dominant contribution in the case of the degenerate spectrum.

For the other mixing channels, it is the induced mass which gives the bound on the parameters of the sterile neutrinos. Indeed, assuming that there is no strong cancellation of elements of the original active neutrino mass matrix $\mathbf{m}_{\mathbf{a}}$ and $\mathbf{m}_{\mathbf{I}}$, and taking the largest elements of matrices in Table 1, we can write the bound

$$
\sin ^{2} \theta_{S} m_{S} \lesssim m^{\exp } \sim(0.5-1) \mathrm{eV}
$$

which is clearly comparable to the neutrinoless double beta decay bound but now valid for all channels. This can be viewed as the forth benchmark line shown in Fig.1-3.

Sterile neutrinos in this range can play some role in leptogenesis and the generation of the baryon asymmetry in the Universe [81, 24].

Clearly contribution from this region is out of our control and this creates ambiguity in the implications of the mass and mixing results.

It is beyond the scope of this paper to discuss the possible origins of $S$ with such a small mixing. Still one possibility looks rather interesting: if $m_{S}=\left(10^{2}-10^{6}\right) \mathrm{GeV}$ (region where one may expect singlets related, e.g., to SUSY breaking), the required mixing is $\sin ^{2} \theta_{S}=10^{-15}-10^{-12}$. The latter can be related to the existence of a new "intermediate" scale $M=m_{S} / \sin \theta_{S} \sim\left(10^{10}-10^{12}\right) \mathrm{GeV}$.

If the interpretation of the LSND result in terms of oscillations in $(3+1)$ scheme is confirmed, that would imply existence of the sterile neutrino(s) with mass $(0.5-5) \mathrm{eV}$ with mixing parameters $\sin ^{2} \theta_{S} \sim 0.02$ (see [82] for recent analysis). The corresponding induced mass equals $m_{I}=(10-100) \mathrm{meV}$ and therefore the effects of LSND neutrino on the active neutrino mass matrix is strong and can not be considered as small perturbation.

2) Low mass window: $m_{S} \sim(0.1-0.3) \mathrm{eV}$ and $\sin ^{2} \theta_{S}=10^{-3}-10^{-1}$. This window is essentially closed for all the channels if one takes the BBN bound $\Delta N_{\nu}<1$. In this case, one has the bound on the induced mass $m_{I}<1 \mathrm{meV}$. That can produce some effect 
on observables in the case of degenerate or inverted hierarchy spectrum. From above this region is restricted by the LSS bound on neutrino mass.

If $\Delta N_{\nu}=1$ is allowed, there is no $\mathrm{BBN}$ bound. Bounds from other effects strongly depend on flavor. For the $\nu_{e}-\nu_{S}$ channel, the reactor and atmospheric neutrino bounds essentially exclude the dominant contribution from $m_{I}$ but still allow for sub-dominant effects. For the $\nu_{\mu}-\nu_{S}$ channel, the bound is given essentially by the atmospheric neutrinos and larger region of mixings is allowed. In particular, for $m_{S} \sim 0.25 \mathrm{eV}$ the mixing $\sin ^{2} \theta_{S}=0.04$ is not excluded leading to $m_{I} \sim 10 \mathrm{meV}$. The latter is close to the dominant contribution. For the $\nu_{\tau}-\nu_{S}$ channel, the atmospheric bound is weaker and dominant contributions from $m_{I}$ are allowed: $m_{I} \sim(30-250) \mathrm{meV}$.

In the rest of the $m_{S}$ region, $m_{S}=\left(10^{-3}-10^{5}\right) \mathrm{keV}$, effects of the direct mixing dominate over the induced matrix effects. In this range the induced masses

$$
m_{I} \lesssim 4 \cdot 10^{-2} \mathrm{meV} \text {, }
$$

can produce only very small corrections to the active neutrino mass matrix. In the interval $m_{S}=\left(1-10^{4}\right) \mathrm{keV}$ the bound is even stronger:

$$
m_{I} \lesssim 10^{-2} \mathrm{meV} .
$$

If however the cosmological/astrophysical bounds are absent for some reason, a large range of parameters becomes allowed and the induced masses can reproduce the dominant structures of the active neutrino mass matrix in the whole range of $m_{S}$.

In Fig. 1 - 3 we show also regions of parameters which correspond to certain positive indications of the existence of sterile neutrinos: (1) overlapping regions of the warm dark matter and pulsar kick, (2) the LSND spot.

In the scenario [24] two neutrinos have masses $m_{S} \gtrsim 1 \mathrm{GeV}$ and their mixing is responsible for the mass matrix of light active neutrinos: $\mathbf{m}_{\mathbf{a}}=\mathbf{m}_{I}$. The third sterile neutrino with mass $m_{S} \sim 1 \mathrm{keV}$ and mixing $\sin ^{2} \theta_{S} \sim 10^{-9}-10^{-8}$ can contribute substantially to warm dark matter (WDM) and explain pulsar kicks. 
Apparently the WDM and pulsar kick regions are far below the benchmark lines and therefore the corresponding induced masses are negligible. Furthermore, the regions are disfavored by the cosmological and astrophysical observations. The WDM scenario can be recovered if the mixing is smaller then that indicated in the plot and some additional mechanism of generation of sterile neutrinos exists apart from mixing with active neutrinos.

In contrast, the LSND spot is in the range where the induced masses are of the order of dominant mass structures. So mixing with sterile neutrino can not be considered as small perturbation of the original active neutrino structure. The LSND spot is essentially excluded by the cosmological data unless some new physics is added.

\subsection{Avoiding bounds}

Let us consider various possibilities which allow one to circumvent the bounds obtained in the previous section and therefore to open a possibility for strong effects of the induced matrix even for low $m_{S}$.

1) It has been shown [60] that in a cosmological scenario with low reheating temperature at the end of inflation, $T_{R}<<100 \mathrm{MeV}$, experimental bounds on the active-sterile neutrino mixing are relaxed. In particular, the sterile neutrino required to explain the LSND result is allowed in this scenario. Also the influence of a non-negligible primordial lepton number asymmetry has impact on the sterile production rate since in this case neutrinos are produced resonantly with a non-thermal spectrum [83, 20, 84]. According to Ref. [20], for $L=10^{-3}$ the LSS limit presented in Figs.1-3 will change very little, while for $L=0.1$ the limit on $\sin ^{2} \theta_{S}$ can increase by 1 or 2 orders of magnitude depending on $m_{S}$.

2) Another possibility can be related to the origin of the mass of $S$ itself. Recall that

$S$ may not be related to right handed neutrinos and usual family structure. Let us assume that $S$ has a "soft mass" generated by the medium dependent VEV of some new scalar field $A$ : $m_{S}=\lambda\langle A\rangle$. The VEV can be proportional to the number density of the active neutrinos, $n_{\nu}:\langle A\rangle \propto n_{\nu}[85]$. In this case

$$
m_{S}=m_{S}^{0}(1+z)^{3}
$$


where $m_{S}^{0}$ is the mass in the present epoch. If the mixing mass is generated by the usual Higgs VEV, which does not change with time, we find that the induced contribution to the light mass and the active-sterile mixing decrease back in time:

$$
\begin{gathered}
m_{I}=\frac{m_{i S}^{2}}{m_{S}^{0}(1+z)^{3}}=m_{I}^{0}(1+z)^{-3}, \\
\sin \theta_{S}=\frac{m_{i S}}{m_{S}^{0}(1+z)^{3}}=\sin \theta_{S}^{0}(1+z)^{-3} .
\end{gathered}
$$

Here $m_{I}^{0}$ and $\theta_{S}^{0}$ are parameters at the present epoch. The combination we plot in Figs. 1-3 changes as

$$
\sin ^{2} \theta_{S} m_{S}=\frac{\sin ^{2} \theta_{S}^{0} m_{S}^{0}}{(1+z)^{3}}
$$

The lines of constant induced mass shift with $z$ to the left - to smaller masses and mixings. This means that in the past all cosmological bounds where satisfied.

Already at the recombination epoch the mass of sterile neutrino becomes of the order $10^{4} \mathrm{GeV}$ for the present mass $m_{S}^{0} \sim 1 \mathrm{eV}$ and the mechanism of oscillation production does not work. Essentially, in this scenario the sterile neutrinos are not produced in the Early Universe and their concentration is negligible. The astrophysical and cosmological bounds we have discussed are not applicable.

3) If $S$ interacts with a massless or low-mass Majoron [86] $\phi$, it can decay invisibly as $S \rightarrow \nu \phi$ or annihilate (see similar mechanism for the active neutrinos in [87]). If this decay is fast enough, $\tau_{S}<<1 \mathrm{~s}$, in principle, all astrophysical and cosmological bounds could be evaded. See [88] for the recent similar analysis. In the mass range $m_{S} \sim 1 \mathrm{keV}$ such a fast decay can be achieved for the scalar coupling $g \sim 10^{-8}$.

In the low mass range some restrictions on the off-diagonal couplings $g \nu_{a} S \phi$ can be obtained from their effect on the free-streeming condition for active neutrinos. The latter should manifest itself in the precision measurements of the CMB acoustic peaks. One can use these bounds also for sterile neutrinos. In [89] a limit on the active neutrino coupling $g \lesssim 1 \cdot 10^{-11}\left(50 \mathrm{meV} / \mathrm{m}_{\mathrm{S}}\right)^{2}$ was obtained. According to Ref. [90], the limit is less severe and is absent if couplings with different active neutrinos are different, e.g., $\mathrm{S}$ has large coupling with $\nu_{\tau}$ only. 


\section{Conclusions}

The main conclusions of this paper can be summarized as follows.

1) Mixing of the active neutrinos with sterile neutrinos, singlets of the SM symmetry group, generates an induced mass matrix of active neutrinos which can be the origin of peculiar properties of the lepton mixing and neutrino mass spectrum. It opens an alternative possibility to understand possible new symmetries in the neutrino sector. In this way, one can explain the substantial difference of mixing patterns of quarks and leptons.

Depending on masses and mixings of $S$, the induced active neutrino masses can be the origin of the dominant or sub-dominant structures of the neutrino mass matrix. For instance, the tri-bimaximal mixing can originate from the induced contribution.

2) Apart from modification of the mass matrix of active neutrinos, there are direct mixing effects of $S$ which can be observed in cosmology, astrophysics and laboratory experiments.

The importance of the direct and induced effects depends on the range of $S$ parameters considered. For $m_{S} \gtrsim 300 \mathrm{MeV}$ the induced effect dominates. The induced masses can reproduce the dominant structures of the active mass matrix. The direct mixing effects are negligible. In the interval $m_{S} \sim\left(10^{-3}-10^{5}\right) \mathrm{keV}$ the direct mixing effects dominate: the astrophysical and cosmological consequences of mixing are more important putting strong upper bounds on the induced mass. So, the latter can be neglected in the mass matrix of active neutrinos. In the narrow window $m_{S} \sim(0.1-0.3) \mathrm{eV}$ the two effects are comparable. For the BBN bound $\Delta N_{\nu}<1$ the induced matrix can produce only small effects. If one additional neutrino is allowed by $\mathrm{BBN}$, the induced masses can generate the sub-leading structures or even be comparable to the values of dominant mass matrix elements.

3) New physics effects can relax or even lift the cosmological and astrophysical bounds thus opening a possibility to generate large induced matrix in the whole range of masses $m_{S}>0.01 \mathrm{eV}$. Here interesting possibilities to notice are: cosmological scenarios with low reheating temperature, fast decay of $S$ into a Majoron and neutrino, and the possibility of a soft mass $m_{S}$ which varies with time, a la Mass Varying Neutrino scenario. 


\section{Acknowledgments}

We are grateful to A. Slosar for proving us with bounds on the energy density of sterile neutrinos in the whole range of masses. We thank U. Seljak for useful discussions and G. G. Raffelt, S. Pastor, A. Kusenko, M. Shaposhnikov and N. Bell for valuable correspondence. This work was partially supported by Fundação de Amparo à Pesquisa do Estado de São Paulo (FAPESP) and Conselho Nacional de Ciência e Tecnologia (CNPq). R.Z.F. acknowledges the Phenomenology Institute of the University of Wisconsin at Madison where the final part of this work was done. 


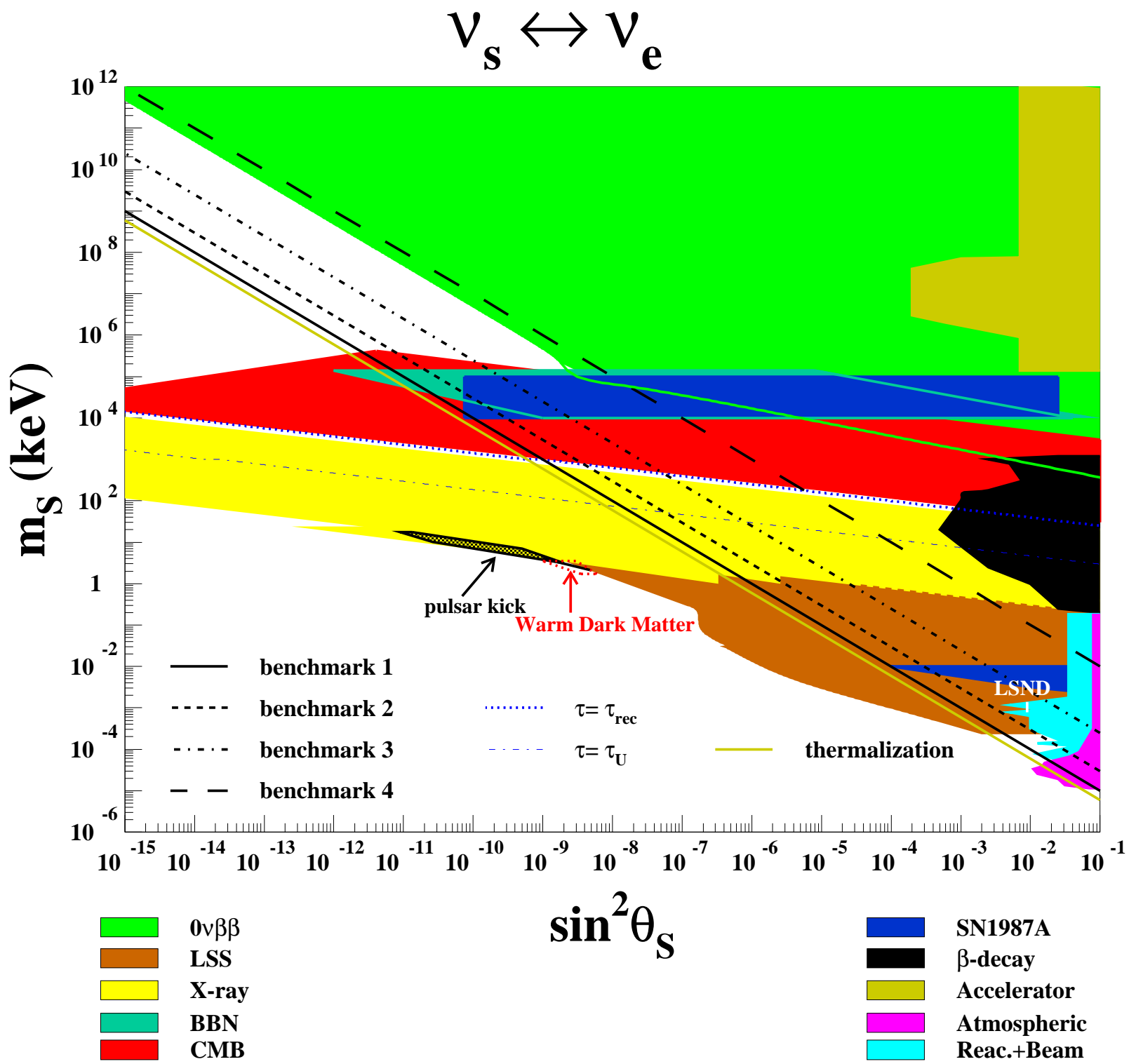

Figure 1: The benchmark lines Eqs. (33)-(35) and (55) versus the current astrophysical, cosmological and laboratory bounds on $\nu_{S}-\nu_{e}$ mixing as described in the text. The colored regions are excluded in each case. The "thermalization" line and the two decay lines $\tau_{S}=\tau_{\text {rec }}$ and $\tau_{S}=\tau_{U}$ are also shown. We show also the allowed regions for the warm dark matter and the LSND $(3+1)$ as well as the region that could explain pulsar velocities. 


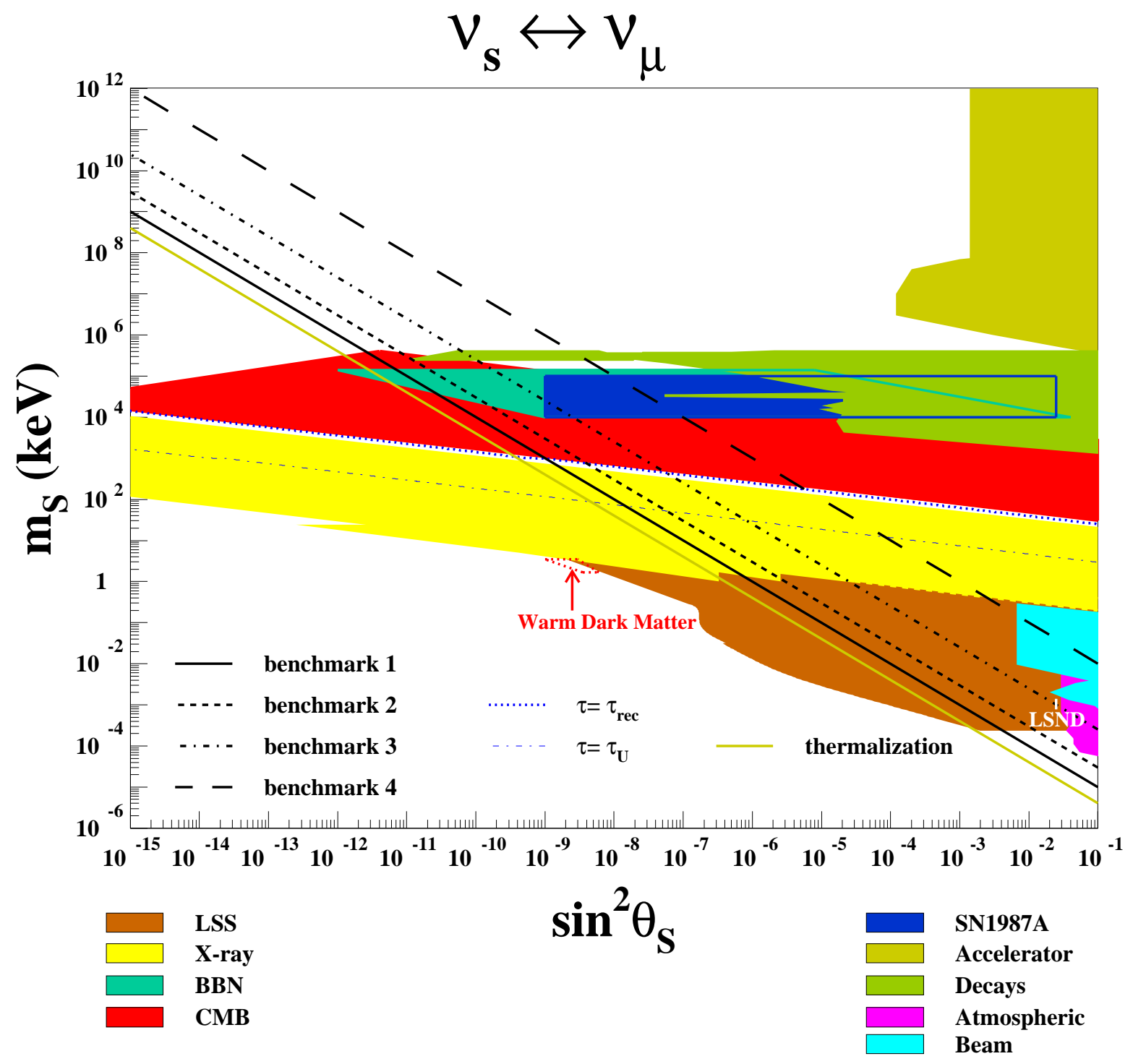

Figure 2: Same as Fig.1 but for $\nu_{S}-\nu_{\mu}$ mixing. 


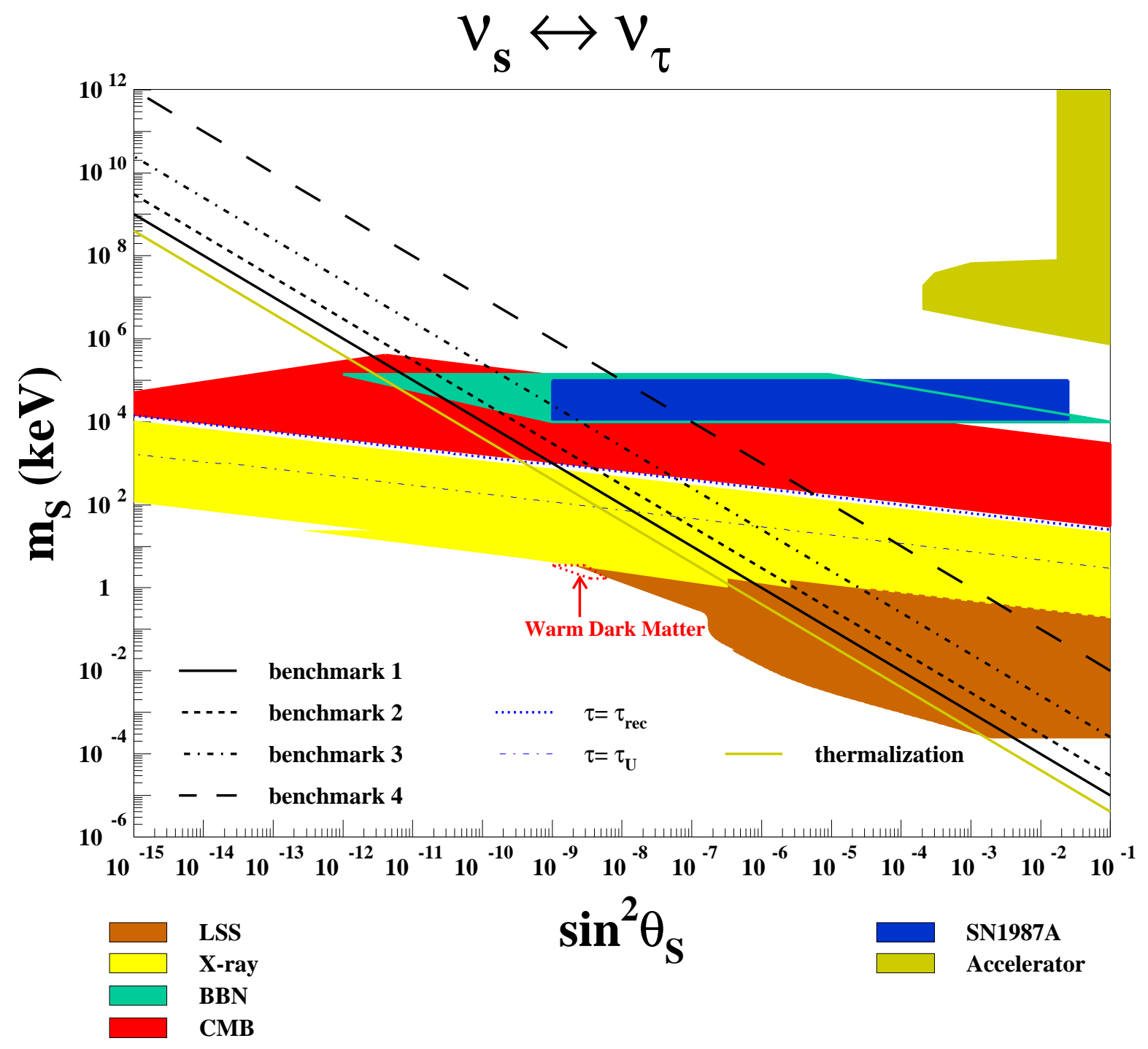

Figure 3: Same as Fig.1 but for $\nu_{S}-\nu_{\tau}$ mixing. 


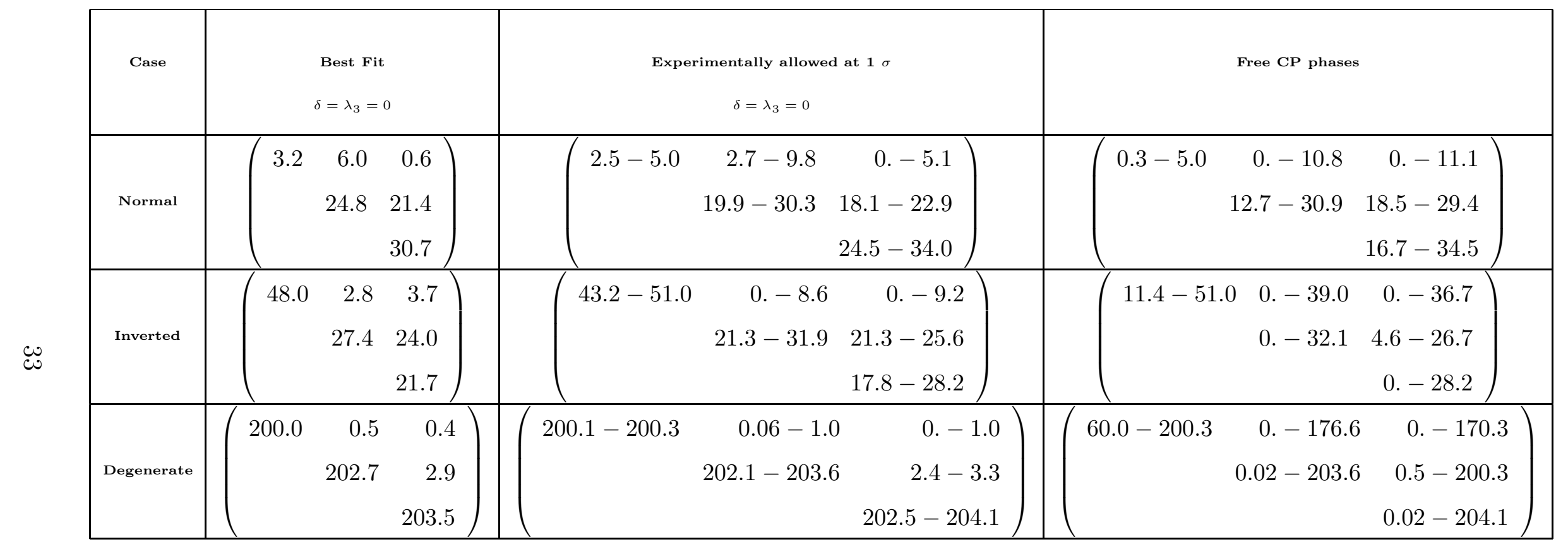

Table 1: The reconstructed matrices for the normal and inverted mass hierarchies as well as the degenerate mass spectrum for $m_{0}=0.2 \mathrm{eV}$. The moduli of elements are given in units of $1 \mathrm{meV}$. For each case we present: the matrix for the best fit values of the mixing parameters, the intervals of the matrix elements obtained varying the experimental values within $1 \sigma$ for $\delta=\lambda_{3}=0$ and also for free CP-violating phases. 


\section{References}

[1] P. Minkowski, Phys. Lett. B 67, 421 (1977); T. Yanagida, Proceedings of the Workshop on The Unified Theory and the Baryon Number in the Universe (O. Sawada and A. Sugamoto, eds.), KEK, Tsukuba, Japan, 1979, p. 95; S. L. Glashow, Proceedings of the 1979 Cargèse Summer Institute on Quarks and Leptons (M. Lévy, J.-L. Basdevant, D. Speiser, J. Weyers, R. Gastmans, and M. Jacob, eds.), Plenum Press, New York, 1980, p. 687; M. Gell-Mann, P. Ramond, and R. Slansky, Supergravity (P. van Nieuwenhuizen and D. Z. Freedman, eds.), North Holland, Amsterdam, 1979, p. 315; R. N. Mohapatra and G. Senjanović, Phys. Rev. Lett. 44, 912 (1980).

[2] P. C. de Holanda and A. Y. Smirnov, Phys. Rev. D 69, 113002 (2004) [arXiv:hepph/0307266]; M. Sorel, J. M. Conrad and M. Shaevitz, Phys. Rev. D 70, 073004 (2004) [arXiv:hep-ph/0305255]; M. C. Gonzalez-Garcia, M. Maltoni and C. Pena-Garay, Phys. Rev. D 64, 093001 (2001) [arXiv:hep-ph/0105269]; W. Grimus and T. Schwetz, Eur. Phys. J. C 20, 1 (2001); C. Giunti and M. Laveder, JHEP 0102, 1 (2001); V. D. Barger et al.,, Phys. Lett. B 489, 345 (2000); S. Goswami, Phys. Rev. D 55, 2931 (1997); J. J. Gomez-Cadenas and M. C. Gonzalez-Garcia, Z. Phys. C 71, 443 (1996).

[3] O. L. G. Peres and A. Y. Smirnov, Nucl. Phys. B 599, 3 (2001) [arXiv:hep-ph/0011054]

[4] M. Cirelli, et al., Nucl. Phys. B 708, 215, (2005).

[5] R. N. Mohapatra and S. Nussinov, Phys. Rev. D 60, 013002 (1999) [arXiv:hep$\mathrm{ph} / 9809415]$.

[6] K. R. S. Balaji, A. Perez-Lorenzana and A. Y. Smirnov, Phys. Lett. B 509, 111 (2001)

[7] J. C. Gomez-Izquierdo and A. Perez-Lorenzana, arXiv:hep-ph/0601223.

[8] A. Aguilar et al. [LSND Collaboration], Phys. Rev. D 64, 112007 (2001) [arXiv:hepex/0104049].

[9] S. Dodelson and L. M. Widrow, Phys. Rev. Lett. 72, 17 (1994). 
[10] See A. D. Dolgov, Phys. Rept. 370, 333 (2002) [arXiv:hep-ph/0202122], for a complete review of references.

[11] A. Kusenko and G. Segre, Phys. Lett. B 396, 197 (1997) [arXiv:hep-ph/9701311]; G. M. Fuller, A. Kusenko, I. Mocioiu and S. Pascoli, Phys. Rev. D 68, 103002 (2003) [arXiv:astro-ph/0307267]. A. Kusenko, Int. J. Mod. Phys. D 13 (2004) 2065 [arXiv:astro-ph/0409521].

[12] P. L. Biermann and A. Kusenko, Phys. Rev. Lett. 96, 091301 (2006) [arXiv:astro$\mathrm{ph} / 0601004]$.

[13] G.L. Fogli, E. Lisi, A. Marrone, A. Palazzo, arXiv:hep-ph/0506083.

[14] A. Y. Smirnov, arXiv:hep-ph/0402264; M. Raidal, Phys. Rev. Lett. 93, 161801 (2004);

H. Minakata and A. Y. Smirnov, Phys. Rev. D 70, 073009 (2004).

[15] K. Enqvist, K. Kainulainen and M. J. Thomson, Nucl. Phys. B 373, 498 (1992).

[16] X. Shi, D. N. Schramm and B. D. Fields, Phys. Rev. D. 48, 2563 (1993).

[17] S. Dodelson, A. Melchiorri and A. Slosar, arXiv:astro-ph/0511500.

[18] A. Slosar, private communication.

[19] K. Abazajian, arXiv:astro-ph/0511630.

[20] K. Abazajian, G. M. Fuller and M. Patel, Phys. Rev. D 64, 023501 (2001).

[21] U. Seljak, A. Solar, and P. McDonald, arXiv:astro-ph/0604335.

[22] U. Seljak, A. Makarov, P. McDonald and H. Trac, arXiv:astro-ph/0602430.

[23] T. Asaka, A. Kusenko and M. Shaposhnikov, arXiv:hep-ph/0602150.

[24] T. Asaka and M. Shaposhnikov, Phys. Lett. B 620, 17 (2005).

[25] T. Asaka, S. Blanchet and M. Shaposhnikov, Phys. Lett. B 631, 151 (2005). 
[26] K. Abazajian, G. M. Fuller and W. H. Tucker, Astrophys. J. 562, 593 (2001).

[27] M. Mapelli and A. Ferrara, Mon. Not. Roy. Astron. Soc. 364, 2 (2005) [arXiv:astro$\mathrm{ph} / 0508413]$.

[28] A. Boyarsky, A. Neronov, O. Ruchayskiy and M. Shaposhnikov, arXiv:astro$\mathrm{ph} / 0603368$.

[29] A. Boyarsky, A. Neronov, O. Ruchayskiy, M. Shaposhnikov, arXiv:astro-ph/0512509.

[30] C. R. Watson, J. F. Beacom, H. Yüksel, and T. P. Walker, arXiv:astro-ph/0605424.

[31] A. D. Dolgov and S. H. Hansen, Astropart. Phys. 16, 339 (2002) [arXiv:hep$\mathrm{ph} / 0009083]$.

[32] P. de Bernardis et al. [Boomerang Collaboration], Nature 404, 955 (2000) [arXiv:astroph/0004404]; A. Balbi et al., Astrophys. J. 545, L1 (2000) [Erratum-ibid. 558, L145 (2001)] [arXiv:astro-ph/0005124].

[33] V. Barger, J. P. Kneller, H. S. Lee, D. Marfatia and G. Steigman, Phys. Lett. B 566, 8 (2003) [arXiv:hep-ph/0305075].

[34] P. Crotty, J. Lesgourgues and S. Pastor, Phys. Rev. D 67, 123005 (2003) [arXiv:astro$\mathrm{ph} / 0302337]$.

[35] E. Pierpaoli, Mon. Not. Roy. Astron. Soc. 342, L63 (2003) [arXiv:astro-ph/0302465].

[36] S. Hannestad, JCAP 0305, 004 (2003) [arXiv:astro-ph/0303076].

[37] S. Hannestad, JCAP 0601, 001 (2006) [arXiv:astro-ph/0510582].

[38] S. Hannestad and G. Raffelt, Phys. Rev. D 59, 043001 (1999) [arXiv:astro-ph/9805223].

[39] D. N. Spergel et al., arXiv:astro-ph/0603449.

[40] R. Bowen, S. H. Hansen, A. Melchiorri, J. Silk and R. Trotta, Mon. Not. Roy. Astron. Soc. 334, 760 (2002) [arXiv:astro-ph/0110636]. 
[41] S. Bashinsky and U. Seljak, Phys. Rev. D 69, 083002 (2004) [arXiv:astro-ph/0310198].

[42] A. D. Dolgov and F. L. Villante, Nucl. Phys. B 679, 261 (2004).

[43] A. D. Dolgov, S. H. Hansen, G. Raffelt and D. V. Semikoz, Nucl. Phys. B 590, 562 (2000) [arXiv:hep-ph/0008138].

[44] K. Hirata et al. [KAMIOKANDE-II Collaboration], Phys. Rev. Lett. 58, 1490 (1987); J. C. Van Der Velde et al. [IMB COLLABORATION Collaboration], Nucl. Instrum. Meth. A 264 (1988) 28.

[45] K. Kainulainen, J. Maalampi and J. T. Peltoniemi, Nucl. Phys. B 358, 435 (1991).

[46] H. Nunokawa, O. L. G. Peres and R. Zukanovich Funchal, Phys. Lett. B 562,279 (2003)

[47] P. Benes, A. Faessler, F. Simkovic and S. Kovalenko, Phys. Rev. D 71, 077901 (2005) [arXiv:hep-ph/0501295].

[48] H. V. Klapdor-Kleingrothaus et al., Eur. Phys. J. A 12, 147 (2001); C. E. Aalseth et al., Phys. Rev. D 65, 092007 (2002).

[49] C. Arnaboldi et al. [CUORE Collaboration], Astropart. Phys. 20 (2003); M. Danilov et al. [EXO Collaboration], Phys. Lett. B 480, 12 (2000); C. E. Aalseth et al. [Majorana Collaboration], Phys. Atom. Nucl. 67, 2002 (2004); H. Ejiri et al. [MOON Collaboration], Phys. Rev. Lett. 85, 2917 (2000); K. Zuber [COBRA Collaboration], Phys. Lett. B 519, 1 (2001); N. Ishihara, T. Ohama and Y. Yamada [DCBA Collaboration], Nucl. Instrum. Meth. A 373, 325 (1996); S. Yoshida et al. [CANDLES Collaboration], Nucl. Phys. Proc. Suppl. 138, 214 (2005); G. Bellini et al. [CAMEO Collaboration], Eur. Phys. J. C 19, 43 (2001); I. Abt et al. [GERDA Collaboration], arXiv:hep-ex/0404039; A. S. Barabash [NEMO Collaboration], arXiv:hep-ex/0602011.

[50] K. Hagiwara et al., Phys. Rev. D 66, 010001 (2002).

[51] D. I. Britton el al., Phys. Rev. D 49, 28 (1994); D. A. Bryman and T. Numao, Phys. Rev. D 53, 558 (1996). 
[52] A. Kusenko, S. Pascoli and D. Semikoz, JHEP 0511, 028 (2005) [arXiv:hep$\mathrm{ph} / 0405198]$.

[53] E. Church et al. [BooNe Collaboration], FERMILAB-PROPOSAL-0898

[54] C. B. Smith [MINOS Collaboration], Int. J. Mod. Phys. A 20, 3059 (2005).

[55] T. Maruyama, Nucl. Instrum. Meth. A 503, 118 (2003).

[56] G. T. Garvey et al., Phys. Rev. D 72, 092001 (2005)[arXiv:hep-ph/0501013].

[57] Y. Fukuda et al. [Super-Kamiokande Collaboration], Phys. Rev. Lett. 81, 1562 (1998) [arXiv:hep-ex/9807003].

[58] M. Ambrosio et al. [MACRO Collaboration], Phys. Lett. B 566, 35 (2003) [arXiv:hepex/0304037].

[59] J. Ahrens et al. [The IceCube Collaboration], Nucl. Phys. Proc. Suppl. 118, 388 (2003) [arXiv:astro-ph/0209556].

[60] G. Gelmini, S. Palomares-Ruiz and S. Pascoli, Phys. Rev. Lett. 93, 081302 (2004) [arXiv:astro-ph/0403323].

[61] G. D'Ali Staiti [EUSO Collaboration], Nucl. Phys. Proc. Suppl. 136 (2004) 415.

[62] Y. Takahashi [OWL Collaboration], Prepared for Ultra High-Energy Cosmic Ray Workshop on Observing Giant Cosmic Ray Air Showers for $>10^{* * 20 ~ e V ~ P a r t i c l e s ~ f r o m ~}$ Space, College Park, MD, 13-15 Nov 199\%.

[63] C. Barbot et al., Phys. Lett. B, 563, 132 (2003).

[64] Y. Declais et al., Nucl. Phys. B 434, 503 (1995).

[65] M. Apollonio et al. [CHOOZ Collaboration], Phys. Lett. B 466, 415 (1999) [arXiv:hepex/9907037].

[66] F. Boehm et al., Phys. Rev. D 62, 072002 (2000) [arXiv:hep-ex/0003022]. 
[67] K. Anderson et al., arXiv:hep-ex/0402041.

[68] V. Kopeikin, L. Mikaelyan, V. Sinev, arXiv:hep-ph/0310246.

[69] F. Dydak et al., Phys. Lett. B 134, 281 (1984).

[70] I. E. Stockdale et al., Phys. Rev. Lett. 52, 1384 (1984).

[71] P. Astier et al. [NOMAD Collaboration], Nucl. Phys. B 611, 3 (2001) [arXiv:hepex/0106102].

[72] E. Eskut et al. [CHORUS Collaboration], Phys. Lett. B 497 (2001) 8.

[73] B. Armbruster et al. [KARMEN Collaboration], Phys. Rev. D 65, 112001 (2002) [arXiv:hep-ex/0203021].

[74] E. Nardi, E. Roulet and D. Tommasini, Phys. Lett. B 327, 319 (1994) [arXiv:hep$\mathrm{ph} / 9402224]$.

[75] O. Adriani et al. [L3 Collaboration], Phys. Lett. B 295, 371 (1992).

[76] Y. Itow et al., arXiv:hep-ex/0106019.

[77] D. S. Ayres et al. [NOvA Collaboration], arXiv:hep-ex/0503053.

[78] A. Ali, A. V. Borisov and N.B. Zamorin, Eur. Phys. J. C21, 123,(2001).

[79] F. del Aguila, J. A. Aguilar-Saavedra, A. Martinez de la Ossa and D. Meloni, Phys. Lett. B 613, 170 (2005) [arXiv:hep-ph/0502189].

[80] F. del Aguila and J. A. Aguilar-Saavedra, JHEP 0505, 026 (2005) [arXiv:hep$\mathrm{ph} / 0503026]$.

[81] E. K. Akhmedov, V. A. Rubakov and A. Y. Smirnov, Phys. Rev. Lett. 81, 1359 (1998) [arXiv:hep-ph/9803255].

[82] S. Goswami and W. Rodejohann, arXiv:hep-ph/0512234. 
[83] X. Shi and G. M. Fuller, Phys. Rev. Lett. 82, 2832 (1999).

[84] K. Abazajian, N. F. Bell, G. M. Fuller and Y. Y. Y. Wong, Phys. Rev. D 72, 063004 (2005) [arXiv:astro-ph/0410175].

[85] R. Fardon, A. E. Nelson and N. Weiner, JCAP 0410, 005 (2004) [astro-ph/0309800].

[86] Y. Chikashige, R.N. Mohapatra, and R.D. Peccei, Phys. Lett. B 98, 265 (1981); J. Schechter and J. W. F. Valle, Phys. Rev. D 25, 774 (1982); G. Gelmini and E. Roulet, Rep. Prog. Phys. 58, 1207 (1995).

[87] J. F. Beacom, N. F. Bell and S. Dodelson, Phys. Rev. Lett. 93, 121302 (2004) [arXiv:astro-ph/0404585].

[88] S. Palomares-Ruiz, S. Pascoli and T. Schwetz, JHEP 0509 (2005) 048.

[89] S. Hannestad and G. Raffelt, Phys. Rev. D 72, 103514 (2005) [arXiv:hep-ph/0509278].

[90] N. F. Bell, E. Pierpaoli, and K. Sigurdson, arXiv:astro-ph/0511410. 